\title{
CFD-based prediction of wall-pressure spectra under a turbulent boundary layer with adverse pressure gradient
}

\author{
Gabriele Grasso • Hao Wu • \\ Susanna Orestano • Marlène Sanjosé • \\ Stéphane Moreau • Michel Roger
}

Received: 30 June 2020 / Revised: 7 October 2020 / Accepted: 10 November 2020

\begin{abstract}
A model of the spectrum of wall-pressure fluctuations under a turbulent boundary layer based on an analytical solution of the Poisson equation is presented. This model is suited for aeroacoustic prediction based on CFDextracted flow information, but requires statistical properties of the boundarylayer turbulence that are not resolved in steady-state simulations and need to be modelled. For this reason, this paper uses Lattice-Boltzmann (DNS-LBM) and Navier-Stokes Direct Numerical Simulations (DNS-NS) of an airfoil in a wind-tunnel jet to investigate the link between turbulence and wall-pressure statistics and validate the assumptions made in the application of the analytical model. The use of input from two numerical simulation methods allows generalizing the results of the analytical model.
\end{abstract}

The reported work is dealing with outcomes of the project SCONE. This project has received funding from the Clean Sky 2 Joint Undertaking under the European Union's Horizon 2020 research and innovation programme under grant agreement No 755543. The authors gratefully acknowledge the support of the Canadian NSERC Discovery grant (no RGPIN2014-04111). This research was enabled in part by support provided by Calcul Québec (www.calculquebec.ca) and Compute Canada (www.computecanada.ca). This work was performed within the framework of the LABEX CeLyA (ANR-10-LABX- 0060) of Université de Lyon, within the programme "Investissements d'Avenir" (ANR-16- IDEX-0005) operated by the French National Research Agency (ANR).

G. Grasso, S. Orestano, M. Roger

Univ Lyon, École Centrale de Lyon, INSA Lyon, Université Claude Bernard Lyon I, CNRS, Laboratoire de Mécanique des Fluides et d'Acoustique, UMR 5509

36 Avenue Guy de Collongue, F-69134, Écully, France

E-mail: gabriele.grasso@ec-lyon.fr

M. Sanjosé

École de Technologie Supérieure, Montréal, QC H3C 1K3, Canada

H. Wu, S. Moreau

Département de Génie Mécanique, Université de Sherbrooke, Sherbrooke, QC, J1K 2R1, Canada 
Keywords Aeroacoustics · turbulent boundary layer · computational fluid dynamics $\cdot$ trailing-edge noise 


\section{Nomenclature}

$\begin{array}{ll}c & \text { Airfoil chord-length } \\ \delta & \text { Boundary layer thickness } \\ \boldsymbol{k}=\left(k_{1}, k_{3}\right) & \text { Planar wavenumber vector } \\ k=\sqrt{k_{1}^{2}+k_{3}^{2}} & \text { Planar wavenumber amplitude } \\ \tilde{k}=k l & \text { Dimensionless wavenumber } \\ \mathrm{K}_{z} & \text { Modified Bessel function of the second kind } \\ l & \text { Turbulence characteristic length scale } \\ M & \text { Mach number } \\ p & \text { Fluctuating pressure variable } \\ T_{c} & \text { Through-flow time over the airfoil } \\ R e_{c} & \text { Reynolds number based on the airfoil chord } \\ u_{i} & \text { Fluctuating velocity component } \\ U_{0} & \text { Wind-tunnel exit velocity } \\ U_{c} & \text { Cnvective speed of wall-pressure fluctuations } \\ U_{e} & \text { External velocity above the boundary layer } \\ \alpha & \text { Ratio of longitudinal to transverse integral length scale } \\ \Lambda & \text { Longitudinal integral length scale of turbulence } \\ \varphi_{p p} & \text { Power spectral density of wall-pressure fluctuations } \\ \varphi_{22} & \text { Cross-spectral density of vertical velocity fluctuations }\end{array}$

\section{Introduction}

Wall-pressure fluctuations under a turbulent boundary layer are a source of both internal and external noise in various means of transportation. On the one hand, structural excitation due to external pressure fluctuations is a significant source of cabin noise. On the other hand, pressure disturbances generated on the surface of several lifting surfaces are scattered into acoustic waves at the trailing edge. Due to the random nature of these disturbances, the generated noise is broadband. Furthermore, the sound power spectral density (PSD) is directly proportional to that of the wall-pressure fluctuations [1].

The aeroacoustic and structural evaluation of cabin comfort concepts makes it necessary to quantify the spectrum of wall-pressure fluctuations under a turbulent boundary layer. Empirical models are often used for this purpose, although their scope of application is limited by the physical conditions of the experiments on which they have been calibrated (see [2] for an extensive review). Many empirical models are calibrated on zero-pressure-gradient boundary layers, therefore they are not able to account for the effect of the adverse pressure gradient that is found in many practical applications. On the contrary, models based on the Poisson equation governing turbulent pressure fluctuations are physically based and therefore more general since they represent the wall-pressure spectrum as a function of certain statistical properties of the turbulent boundary layer. For this reason, they are suitable for the predic- 
tion of wall-pressure statistics given boundary-layer characteristics extracted from CFD simulations. Inhomogeneity and anisotropy of the turbulence are most of the time neglected in the RANS approach selected in industrial practice. Therefore, the fundamental problem in the application of the analytical wall-pressure PSD model with steady-state simulation input consists of modelling the two-point statistics of the turbulent velocity fluctuations across the boundary layer, taking into account inhomogeneity and anisotropy effects, as shown in the early attempts of [3] and [4]. It may be mentioned that the TNO-Blake family of models is based on an approximate solution of the Poisson equation (see [5] for a review), though there are serious concerns about the physical consistency of the approximation, as discussed in [6].

This work is mainly based on the analytical framework developed in [6], which has been validated by means of Direct Numerical Simulation of the Navier-Stokes equations (DNS-NS). This is certainly the most accurate flow simulation technique, but it has a very high computational cost. LatticeBoltzmann Method Direct Numerical Simulation (DNS-LBM) also has high predictive capabilities, especially in the case of low-Mach number flows, at a lower computational cost. In this work, the input data to the wall-pressure PSD model extracted from DNS-NS and DNS-LBM simulation will be compared in order to generalize the assumptions made in the development of the model, continuing the work of [7]. Finally, it will be shown that the application of the analytical model to data extracted from the two numerical simulations leads to consistent predictions of the wall-pressure PSD, which also compare satisfactorily with measurements.

\section{Analytical formulation}

The following discussion summarizes the main steps of the derivation of the analytical framework, which is fully detailed in [6].

Taking the divergence of the incompressible momentum equation, introducing Reynolds decomposition into mean and fluctuating quantities and then subtracting the time-averaged equation yields the Poisson equation governing the pressure fluctuations in a turbulent boundary layer. In the incompressible flow limit and in a non-rotating frame of reference (see [8]), the equation reads

$$
\frac{1}{\rho} \nabla^{2} p=\underbrace{-2 \frac{\partial u_{j}}{\partial x_{i}} \frac{\partial U_{i}}{\partial x_{j}}}_{\text {turbulence-mean shear }} \underbrace{-\frac{\partial^{2}}{\partial x_{i} \partial x_{j}}\left(u_{i} u_{j}-\overline{u_{i} u_{j}}\right)}_{\text {turbulence-turbulence }}
$$

where the Laplacian of pressure fluctuations is equal to the sum of two source terms. The subscripts $i, j=1,2,3$ correspond to the coordinate system represented in Fig. 1. For the sake of simplicity, it can be assumed that the leadingorder contribution to the wall-pressure spectrum is that of the turbulencemean shear interaction source term, following the analytical considerations of [9] and [10]. Furthermore, assuming that the only non-vanishing mean velocity gradient is that of the streamwise component, $U_{1}$, in the wall-normal 


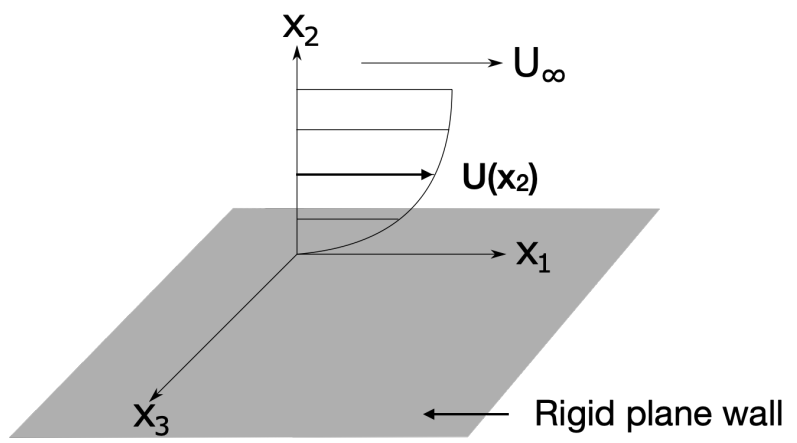

Fig. 1 Frame of reference for the solution of Poisson's equation.

direction, $x_{2}$, and that the turbulence is homogeneous in planes parallel to the wall, Eq. (1) can be Fourier transformed in the $\left(x_{1}, x_{3}\right)$ directions, yielding the following modified Helmholtz equation:

$$
\frac{\partial^{2} \hat{p}\left(\boldsymbol{k}, x_{2}\right)}{\partial x_{2}^{2}}-k^{2} \hat{p}\left(\boldsymbol{k}, x_{2}\right)=-2 \rho \text { i } k_{1} \frac{\partial U_{1}}{\partial x_{2}} \hat{u}_{2}\left(\boldsymbol{k}, x_{2}\right) .
$$

Introducing the boundary conditions on the fluctuating pressure

$$
\lim _{x_{2} \rightarrow \infty} p=0
$$

and

$$
\left.\frac{\partial p}{\partial x_{2}}\right|_{x_{2}=0}=0
$$

the unsteady pressure has the following solution in the wavenumber domain and for $x_{2}=0$ :

$$
\hat{p}(\boldsymbol{k})=2 \mathrm{i} \rho \frac{k_{1}}{k} \int_{0}^{\infty} \frac{\partial U_{1}\left(X_{2}\right)}{\partial x_{2}} \hat{u}_{2}\left(\boldsymbol{k}, X_{2}\right) \mathrm{e}^{-k X_{2}} \mathrm{~d} X_{2}
$$

Multiplying Eq. (3) by its complex conjugate and taking the ensemble average of the product yields the following expression of the wall-pressure PSD generated by the turbulence-mean shear source term:

$\varphi_{p p}^{T M}(\boldsymbol{k})=4 \rho_{0}^{2} \frac{k_{1}^{2}}{k^{2}} \iint_{0}^{\infty} \mathrm{e}^{-\left(X_{2}+X_{2}^{\prime}\right) k} \frac{\partial U_{1}\left(X_{2}\right)}{\partial x_{2}} \frac{\partial U_{1}\left(X_{2}^{\prime}\right)}{\partial x_{2}} \varphi_{22}\left(\boldsymbol{k}, X_{2}, X_{2}^{\prime}\right) \mathrm{d} X_{2} \mathrm{~d} X_{2}^{\prime}$. 
The term $\varphi_{22}$ in the previous equation is the cross-spectral density (CSD) of vertical velocity fluctuations, which is not computed in steady-state simulations. The most general analytical model of $\varphi_{22}$ - the generalized von Kármán energy spectrum - is given in [11] and [12] within the hypothesis of homogeneous and isotropic turbulence as

$$
\varphi_{22}\left(\boldsymbol{k}, x_{2}, x_{2}^{\prime}\right)=\frac{\sqrt{\overline{u_{2}^{2}}\left(x_{2}\right) \overline{u_{2}^{2}}\left(x_{2}^{\prime}\right)} l^{2} \tilde{k}^{2} \zeta^{\nu+2}}{\Gamma(\nu) \pi 2^{\nu+1}\left(1+\tilde{k}^{2}\right)^{\nu+2}} \mathrm{~K}_{\nu+2}(\zeta)
$$

with the parameter

$$
\zeta=\frac{\left\|x_{2}-x_{2}^{\prime}\right\|}{l} \sqrt{1+\tilde{k}^{2}} .
$$

and the characteristic length scale

$$
l=\frac{\Gamma(\nu)}{\sqrt{\pi} \Gamma(\nu+1 / 2)} \Lambda .
$$

The advantage of this formulation is in its flexibility. In fact, for $\nu=1 / 3$, it corresponds to the model of von Kármán [13]; for $\nu=1 / 2$ to Liepmann's model [14] and, for $\nu=7 / 6$ to the rapid distortion theory (RDT) [15]. The effectiveness of these models in describing the statistics of boundary-layer turbulence and predicting the wall-pressure PSD has been discussed at length in $[6]$.

The anisotropy of turbulence length scales across the boundary layer is taken into account by means of the parameter $\alpha\left(x_{2}\right)$, which is the ratio of the longitudinal to transverse integral length scales. Following [16], we define the anisotropic (NI) turbulence CSD as the following function of the isotropic (I) formulation:

$$
\varphi_{22}^{N I}\left(k_{1}, k_{3}, x_{2}, x_{2}^{\prime}\right)=\alpha \varphi_{22}^{I}\left(\alpha k_{1}, k_{3}, x_{2}, x_{2}^{\prime}\right),
$$

where we can take in first approximation $\alpha=\left(\alpha\left(x_{2}\right)+\alpha\left(x_{2}^{\prime}\right)\right) / 2$. Likewise, the integral length scale appearing in Eq. (7) is calculated as $\Lambda=\left(\Lambda\left(x_{2}\right)+\right.$ $\left.\Lambda\left(x_{2}^{\prime}\right)\right) / 2$. Furthermore, we will assume that the wall-pressure PSD at a given radian frequency $\omega$ is made up of gusts convected along the mean-flow direction at the velocity $U_{c}$ according to Taylor's hypothesis [17], whereby the gusts have a unique streamwise wavenumber $K_{c}=\omega / U_{c}$. Therefore, the frequency and wavenumber spectra are linked by the following equation:

$$
\varphi_{p p}^{T M}(\omega)=\frac{\int_{-\infty}^{+\infty} \varphi_{p p}^{T M}\left(K_{c}, k_{3}\right) \mathrm{d} k_{3}}{U_{c}} .
$$

Finally, it is worth reminding that the derivation of a wavenumber solution for the component of the wall-pressure PSD generated by turbulence-turbulence interaction is more cumbersome and requires a set of more restrictive hypotheses in order to get a simple closed-form expression, as detailed in [6]. This component, however, is supposed to be of secondary importance [3]. 


\section{Prediction of wall-pressure spectra}

\subsection{Test case}

Controlled-diffusion (CD) airfoils are conceived so as to carefully control flow and losses close to the airfoil surface [18]. They are designed analytically to be shock-free at transonic Mach numbers and to avoid boundary layer separation on the suction side for a range of inlet conditions typical of compressor applications. Characterized by $4 \%$ thickness to chord ratio and camber angle equal to $12^{\circ}$, the present CD profile has been used for turbo-engine compressor and fan blades and automotive engine cooling fan systems, for instance. The CD profile shape tends to have thicker leading and trailing edges than its standard series counterparts, which lead to improved compressor durability.

The CD airfoil has been extensively investigated experimentally and numerically as a dedicated configuration for the study of trailing-edge broadband noise for the past twenty years [19]. The mock-up is equipped with Remote Microphone Probes (RMP) [20] to measure the wall pressure fluctuations, as shown in Fig. 2. In the present work, we will focus on the prediction of the wallpressure PSD at a point of the suction side corresponding to the RMP\#22, highlighted in red in Fig. 2. This probe is chosen because it is located on the part of the suction side characterized by adverse pressure gradient. The neighbouring probes \#21 and \#24 have been the object of the analysis conducted in [6] on the basis of the DNS-NS simulation alone. The wall-pressure PSD is measured in the anechoic open-jet wind tunnel of the Universite de Sherbrooke (see [21]). In this configuration, the airfoil of chord length $c=0.1356 \mathrm{~m}$ is located in the middle of the air stream of speed $U_{0}=16.0 \mathrm{~m} / \mathrm{s}$ with an angle of attack of $8^{\circ}$ at ambient conditions. This corresponds to a Reynolds number based on the chord $R e_{c}=1.5 \times 10^{5}$ and a Mach number $M=0.05$.

The measured spectrum will be compared with the spectra resulting from the analytic approach of Eq. (4). The required inputs are the tangential velocity gradients across the boundary layer and the cross-spectral density function of the normal velocity fluctuations above RMP\#22. The latter is not available from experimental measurements and can be modeled under the strong hypothesis of Eq. (5). On the other hand, the CSD can be computed from fully resolved turbulent simulations. In this study, the DNS results are obtained from a high-order compressible Navier-Stokes (NS) solver HiPSTAR [22] and from a Lattice-Boltzmann Method (LBM) commercial solver PowerFLOW (v5.1a) [23]. They are used to provide the analytical approach all the necessary statistical inputs in the boundary layer developing above RMP\#22 without further assumptions. In addition, the wall-pressure fluctuation spectra computed from the DNS databases will also be used for the validation of the analytical approach.

The numerical setups of the DNS-NS and of the DNS-LBM simulations are thoroughly described in [24] and [25], respectively. The two configurations mimic the exact same installation of the airfoil in the open-jet wind-tunnel of width $50 \mathrm{~cm}$ (see [21]). Only a span of $10 \% c$ is computed and the Mach number 
has to be increased, to limit both the numerical errors and the computational grid with the employed compressible numerical methods. The Mach number selected for the simulations is $M_{L B M}=0.22$ and $M_{N S}=0.25$ for the DNSLBM and DNS-NS respectively. Note, that the Reynolds number is kept equal to the experimental one $R e_{c}$ by modifying the transport properties of the air medium. In the DNS-LBM, the jet shear layers are resolved in time, yielding to low frequency fluctuations on the airfoil loading, while in the DNS-NS the jet loading effect is provided through the velocity profiles extracted from a previous RANS simulation applied on the domain boundary conditions. For that reason, the statistics are acquired on a longer time corresponding to 10 characteristic times $T_{c}=c / U_{0}$ on the DNS-LBM, while they are acquired on $7 T_{c}$ for the DNS-NS simulation.

For informative purposes, the run time required by the two simulations is presented in Table 2. The reported run time are extracted from the actual performed jobs with all data exports enabled and are not from optimized configurations but from typical solver usages. They are measured on the exact same cluster Graham, which is located at the University of Waterloo managed by Sharcnet and Compute Canada. The computing nodes are Intel Xeon Processor E5-2683 v4 (Broadwell, 2017) and gathered in 1024 cores non-blocking partition with a EDR $(100 \mathrm{~Gb} / \mathrm{s})$ InfiniBand interconnect and are efficiently connected to storage disks with a low-latency. From values provided in Table 2, the DNS-NS must run for 193 hours on 1024 cores (32 nodes) of Graham to simulate 1 convective time $T_{c}$, while it takes only 8.3 hours for the DNS-LBM.

\begin{tabular}{c|c|c|c} 
Simulation & \# of elements & Reduced Efiiciency & Timestep \\
\hline DNS-NS & $345 \mathrm{M}$ cells & $1.5 \times 10^{-5} \mathrm{~s}-\mathrm{cpu} /$ ite $/ \mathrm{elm}$ & $6.4 \times 10^{-8} \mathrm{~s} /$ ite \\
DNS-LBM & $640 \mathrm{M}$ voxels & $6.1 \times 10^{-7} \mathrm{~s} / \mathrm{cpu} /$ ite/elm & $1.1 \times 10^{-7} \mathrm{~s} /$ ite
\end{tabular}

Table 2 Comparison of simulation time.

Figure 3 compares the pressure coefficient calculated by means of the two simulation techniques with the wind-tunnel measurement. In this case, the DNS-LBM simulation is closer to the experimental data because it resolves the full wind-tunnel installation (not only the mean jet deflection). Along the airfoil suction side a turbulent boundary layer develops which is naturally triggered by a small and thin laminar bubble recirculation that appears close to the leading edge and is noticeable by the plateau in the $C_{p}$ profiles (Fig. 3). The size of the bubble is slightly different in the two simulations providing a small deviation in the turbulent state of the boundary layer captured at RMP\#22 in both simulations. Comparisons of DNS-NS, DNS-LBM and experimental boundary-layer mean velocity profiles corresponding to various RMPs are presented in [26, Fig. 8]. Comparisons of the mean wake-velocity profiles can also be found in [26, Fig. 9]. Finally, comparisons of DNS-NS and experimental mean and RMS wake-velocity profiles are shown in [27, Figs. 3-4]. 


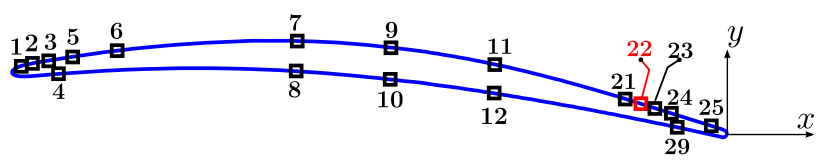

Fig. 2 Distribution of wall-pressure sensors on the surface of the CD airfoil.

\begin{tabular}{crr} 
& DNS-LBM & DNS-NS \\
\hline$\delta_{99}[\mathrm{~mm}]$ & 4.69 & 4.94 \\
$\mathrm{H}$ & 1.76 & 1.89 \\
$U_{e}[\mathrm{~m} / \mathrm{s}]$ & 18.20 & 17.40 \\
\hline
\end{tabular}

Table 1 DNS-NS and DNS-LBM boundary layer parameters.

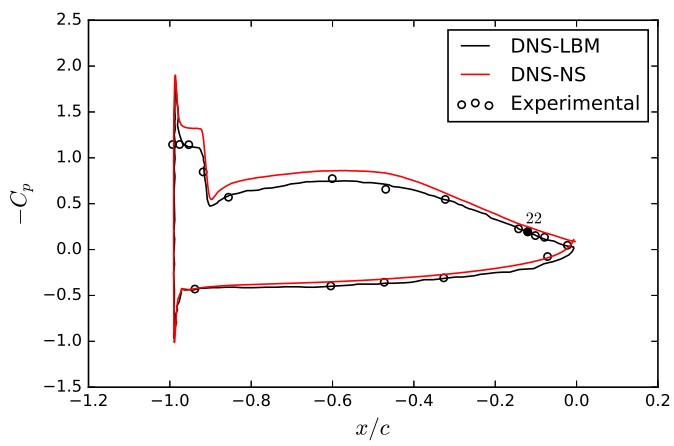

Fig. 3 Comparison of the pressure coefficient extracted from DNS-NS and DNS-LBM simulations over the CD airfoil surface and of the corresponding wind-tunnel measurement.

\subsection{Model input data}

According to the theory detailed in Sec. 2, five different input data are necessary for the prediction of the wall-pressure spectrum. The data are the following:

- the mean streamwise velocity $U_{1}\left(x_{2}\right)$;

- the mean-squared vertical velocity fluctuation, $\overline{u_{2}^{2}}\left(x_{2}\right)$;

- the longitudinal integral length scale, $\Lambda\left(x_{2}\right)$;

- the ratio of longitudinal to transverse length scales, $\alpha\left(x_{2}\right)$;

- the cross-spectral density of vertical velocity fluctuations, $\varphi_{22}\left(k_{1}, k_{3}, x_{2}, x_{2}^{\prime}\right)$.

The boundary layer profiles of $U_{1}\left(x_{2}\right)$ are compared in Fig. 4. The boundary layer is thinner in the DNS-LBM case: this is due to a slightly thinner leadingedge recirculation bubble than in the DNS-NS case. However, the shape factor reported in Table 1 does not vary significantly (as can be expected for a fully turbulent velocity profile). Figure 5 compares the profiles of mean squared vertical velocity fluctuation. This plot confirms that the DNS-LBM predicts a 
thinner boundary layer with a slightly lower turbulence intensity, as a consequence of the leading-edge recirculation bubble.

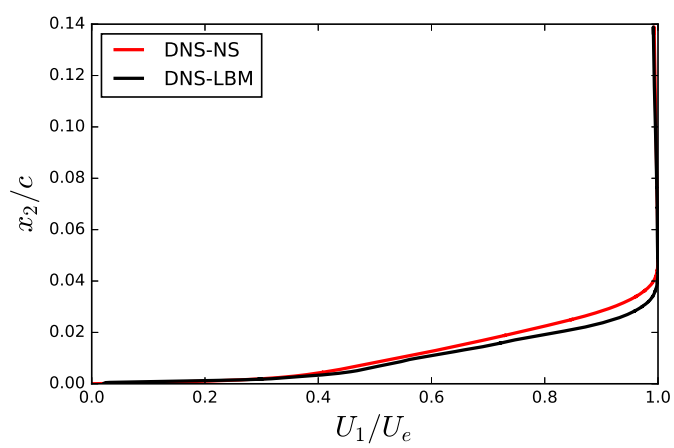

Fig. 4 Comparison of the streamwise component of the mean velocity extracted from DNSNS and DNS-LBM simulations corresponding to the sensor 22 of the CD airfoil.

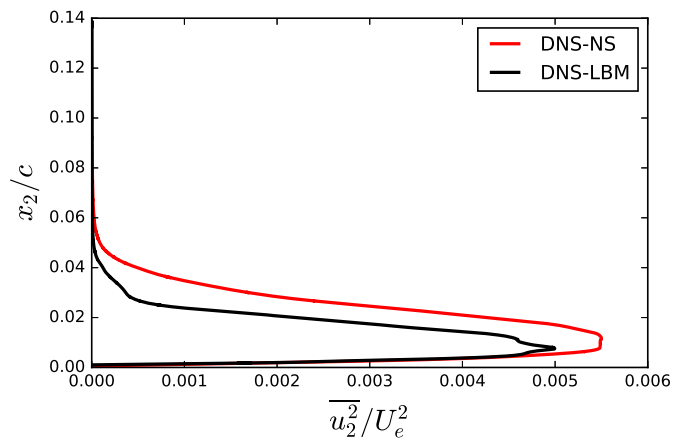

Fig. 5 Comparison of the mean squared vertical velocity fluctuation extracted from DNSNS and DNS-LBM simulations corresponding to the sensor 22 of the CD airfoil.

The longitudinal integral length scale, $\Lambda$, is defined as the integral of the longitudinal correlation function, $F(r)$, depicted in Fig. 6. The CFD-based curves are compared with the analytical generalized von Kármán formulation

$$
F(r)=\frac{1}{2^{\nu-1} \Gamma(\nu)}\left(\frac{r}{l}\right)^{\nu} \mathrm{K}_{\nu}\left(\frac{r}{l}\right)
$$

where the values $\nu=7 / 6$ and $\nu=1 / 3$, corresponding to the RDT and the von Kármán formulations respectively, have been used. It can be seen that the first model is in better agreement with the CFD data. The longitudinal correlation function remains slightly higher than zero for large separations in the DNS-LBM case. This is due to the interpolation of the velocity field on 
planes parallel to the wall. In fact, in the DNS-LBM simulation the Cartesian mesh is not aligned with the airfoil wall and an interpolation must be made to collect and compute statistics. Therefore, the integration for the computation of $\Lambda$ from the DNS-LBM correlation function is truncated. The computed $\Lambda$ profiles are shown in Fig. 7. They are in close agreement in the vicinity of the wall. Further away, however, $\Lambda_{L B M}$ is larger than $\Lambda_{N S}$. Further experimental investigation is needed to clarify this discrepancy.

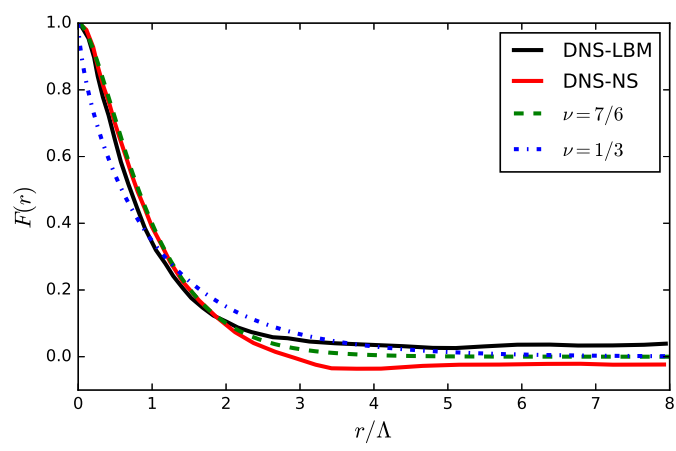

Fig. 6 Comparison of the longitudinal correlation function, $F(r)$, extracted from DNS-NS and DNS-LBM simulations. The dashed line corresponds to the analytical formulation of Eq. (10).

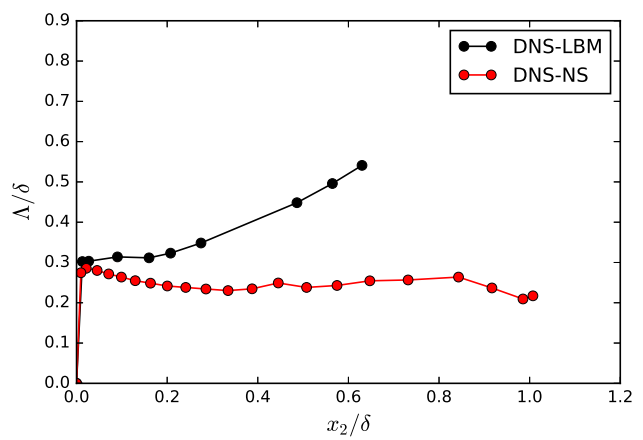

Fig. 7 Comparison of the longitudinal integral length scale, $\Lambda$, extracted from DNS-NS and DNS-LBM simulations.

The anisotropy coefficient, $\alpha$, is plotted for the two simulations in Fig. 8. Its evolution in the normal-to-wall direction remains the same. The slight shift of the plot is due to the different thickness of the boundary layer in the two configurations. 


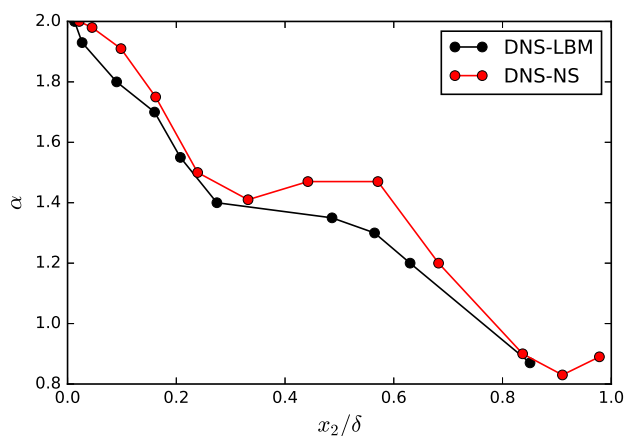

Fig. 8 Comparison of the ratio of length scales, $\alpha$, extracted from DNS-NS and DNS-LBM simulations.

The vertical velocity correlation coefficient, $R_{22}$, is plotted for the two simulation techniques against transverse separations in Figs. 9 and 10, for two different distances from the wall. The vertical-velocity CSD, $\varphi_{22}$, is the double spatial Fourier transform of $R_{22}$, as detailed in [6]. The CFD-based curves are compared with the analytical formulation

$$
\begin{aligned}
R_{22}\left(r_{3}\right)= & \frac{1}{2^{\nu-1} \Gamma(\nu)}\left(\frac{\left|r_{3}\right|}{l}\right)^{\nu} \\
& \times\left[(\nu+1) \mathrm{K}_{\nu}\left(\frac{\left|r_{3}\right|}{l}\right)-\frac{1}{2}\left(\frac{\left|r_{3}\right|}{l}\right) \mathrm{K}_{\nu+1}\left(\frac{\left|r_{3}\right|}{l}\right)\right]
\end{aligned}
$$

given in [11], again with $\nu=7 / 6$ and $\nu=1 / 3$. The reference point at the centre of the domain is the same for DNS-LBM and DNS-NS. Both at a small and at a large distance from the wall, an overall agreement can be found between the results of the two simulations. DNS-LBM simulation data capture well the peak and the negative lobes of the correlation. However, a small difference is noted in the symmetry of the lobes of the $R_{22}$ curves. At $x_{2}^{+}=6$, Fig. 9 , the computed correlation coefficient shows a better match with the von Kármán formulation, especially for small transverse separations. At $x_{2}^{+}=130$, on the contrary, there is a better agreement with the RDT. This result is consistent with the analysis of the DNS results conducted in [6]. The use of the RDT is also consistent with the finding of Magnaudet (see [28]) that this theory is the leading-order approximation describing the short-term and long-term evolution of turbulent boundary layers in the limit of large Reynolds number.

\subsection{Application of the analytical model}

The last step of this study concerns the prediction of the wall-pressure PSD on the basis of the DNS-NS and DNS-LBM input data. The boundary layer volumes around RMP\#22 extracted from the simulations are around $10 \%$ chord in the streamwise direction with all spanwise points taken into account. 


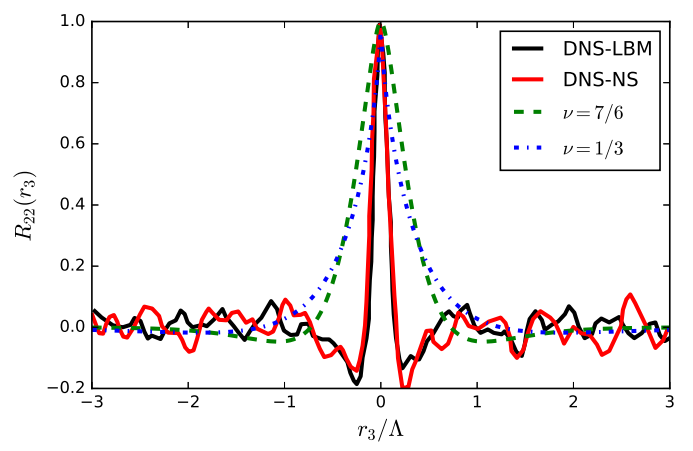

Fig. 9 Comparison of the vertical velocity correlation coefficients, $R_{22}$, extracted from DNS-NS and DNS-LBM simulations at a plane with $x_{2}^{+}=6$. The dashed line corresponds to the analytical formulation of Eq. (11).

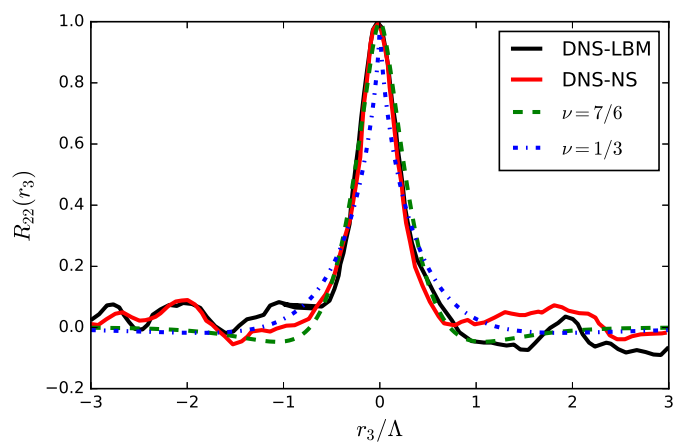

Fig. 10 Comparison of the vertical velocity correlation coefficients, $R_{22}$, extracted from DNS-NS and DNS-LBM simulations at a plane with $x_{2}^{+}=130$. The dashed line corresponds to the analytical formulation of Eq. (11).

The wall-surface spatial resolution is $\Delta x=\Delta y \approx 80 \mu \mathrm{m}$ in the DNS-NS. In the DNS-LBM, the minimum cubic volume cell has size $\Delta x=15 \mu \mathrm{m}$ close to the airfoil surface, but is not body fitted. Hence the temporal quantities are interpolated on a cartesian grid with a spatial resolution of $\Delta x=\Delta y \approx$ $60 \mu \mathrm{m}$ in both streamwise and spanwise directions with a weighted-distance interpolation using 9 points. The prediction is achieved by means of Eqs. (4), (5) and (9). In both cases, the predicted spectra are compared with the ones computed directly from the pressure field of the respective CFD simulations and with the measured spectrum. Consistently with the results presented in [6], the RDT turbulence model provides the most accurate representation of the turbulence statistics across the boundary layer of the CD airfoil in the rear part of the suction side. For this reason, the vertical velocity CSD is predicted by imposing $\nu=7 / 6$ in Eq. (5). The wall-pressure PSDs based on the DNSNS and DNS-LBM simulations are shown in Figs. 11 and 12, respectively. The results obtained with $\nu=1 / 3$ are also shown to highlight the influence of the 
turbulence model. It has been shown in [6] that the wall-pressure PSD in the frequency range of interest is determined by the isotropic part of the boundary layer, therefore the predictions are made by assuming $\alpha=1$.

For the computation of the PSDs from the wall-pressure time series, a periodogram technique is applied. The full time signal is split into windows of $0.77 T_{c}$ with $50 \%$ overlap and a Hanning windowing function is applied. It may be noticed that the DNS-NS simulation yields a wall-pressure PSD closer to the experiment than the DNS-LBM at high frequencies. On the contrary, the DNS-LBM is more accurate at low frequencies because it takes into account the installation effects. Overall, the PSD modelled on the basis of the DNS-LBM boundary-layer profiles is slightly closer to the experimental curve than the one based on DNS-NS data. The only major difference between the boundary layer profiles predicted by the two simulations is in the level of $\Lambda$ in the outer boundary layer, as shown in the previous section. The fact that this does not have a major influence on the predicted wall-pressure PSD is probably explained by the exponential decrease of the integrand of Eq. (4) with the distance from the wall, so that for any given frequency a higher weight is given to the near-wall physics. In any case, both modelled spectra approximate well the experimental data from $400 \mathrm{~Hz}$ to $10 \mathrm{kHz}$. Below $400 \mathrm{~Hz}$ the measured and directly computed spectra exhibit a plateau. On the contrary, in this range of frequencies the modelled spectra increase proportionally to $\omega^{2}$. It has been shown in [6] that the quadratic rise of the wall-pressure PSD in the low frequency range is an inherent feature of all models based on the Poisson equation. Empirical models such as those of Goody [29] and Rozenberg [30] also take into account this quadratic rise. As discussed in [6], the difference is partly due to the fact that some significant effect from the jet is unavoidable both in the experiment and in the numerical simulations (especially the DNS-LBM, which also contains the jet unsteadiness), which prevents us from obtaining the theoretical positive slope in the wall-pressure spectra. Also, the hump around $1000 \mathrm{~Hz}$ is due to the slight vortex shedding (an illustration of this mechanism can be seen in Fig. 10 (a) in [31]) on the pressure side, an effect that is not taken into account by the analytical model . Finally, the current wallpressure PSD predictions are obtained by assuming that the convection speed of wall-pressure fluctuations, $U_{c}$, is constant with respect to the frequency (see Eq. (9)). In this case, it has been assumed that $U_{e} / U_{c}=1.51$, consistently with the results of [6]. This is valid in first approximation, but in a future work the convective speed will be calculated directly from the CFD data as a function of frequency and that could possibly alter the predicted PSD especially at low frequencies.

\section{Conclusions and perspectives}

Both the DNS-NS and the DNS-LBM simulations provided input data to the analytical model that resulted in a satisfactory prediction of the wall-pressure PSD. Despite the inherent discrepancies between the two numerical simula- 


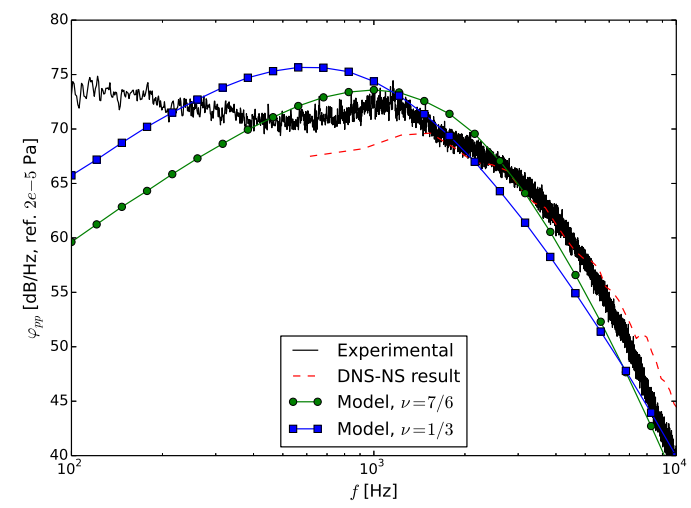

Fig. 11 Comparison of the wall-pressure PSD measured, directly computed from DNS-NS and modelled using the corresponding boundary layer profiles.

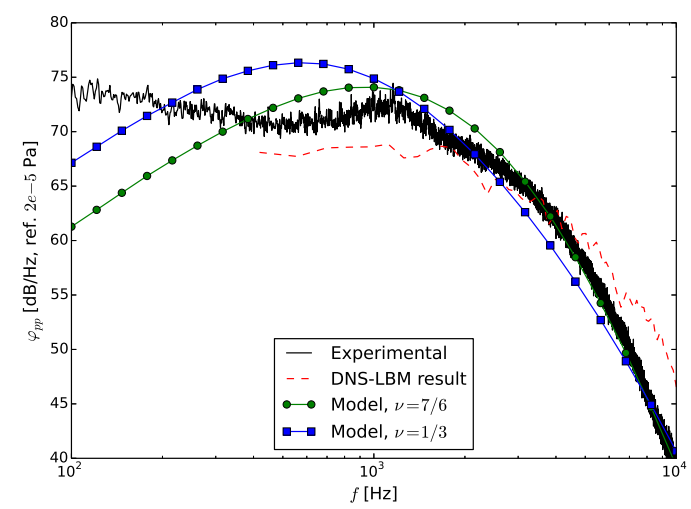

Fig. 12 Comparison of the wall-pressure PSD measured, directly computed from the DNSLBM simulation and modelled using the corresponding boundary layer profiles.

tion methods, we obtain consistent modeled wall-pressure PSD predictions, thus confirming the robustness of the analytical method. The interest of using these high-fidelity simulations is that they allow to compute directly some statistical properties of the boundary-layer turbulence that need be modelled if a steady-state simulation is used to provide the input data. Therefore, the results presented in this work provide guidelines for the application of the analytical model to steady-state simulation input, which is the least computationally expensive way to predict the wall-pressure PSD.

The analytical model of wall-pressure PSD based on the Poisson equation has been applied to a test case in incompressible flow regime. The extension of the model to the compressible flow regime, in order to apply it to a wider range of industrial cases especially at high Mach number, is currently under investigation. 
Acknowledgements This project has received funding from the Clean Sky 2 Joint Undertaking under the European Union's Horizon 2020 research and innovation programme under grant agreement No 755543

\section{Conflict of interest}

The authors declare that they have no conflict of interest.

\section{References}

1. R. K. Amiet. Noise due to turbulent flow past a trailing edge. Journal of Sound and Vibration, 4(3), 1976.

2. S. Lee. Empirical wall-pressure spectral modeling for zero and adverse pressure gradient flows. AIAA Journal, 56(5):1818-1829, 2019/02/11 2018.

3. R. L. Panton and J. H. Linebarger. Wall Pressure Spectra Calculations for Equilibrium Boundary Layers. Journal of Fluid Mechanics, 65(02):261-287, 1974.

4. S. Remmler, J. Christophe, J. Anthoine, and S. Moreau. Computation of wall-pressure spectra from steady flow data for noise prediction. AIAA Journal, 48(9):1997-2007, 2010.

5. M. Kamruzzaman, Th. Lutz, W. Würz, W. Z. Shen, W. J. Zhu, M O. L. Hansen F. Bertagnolio, and H. Aa. Madsen. Validations and improvements of airfoil trailingedge noise prediction models using detailed experimental data. Wind Energy, 15:45-61, 2012.

6. G. Grasso, P. Jaiswal, H. Wu, S. Moreau, and M. Roger. Analytical models of the wallpressure spectrum under a turbulent boundary layer with adverse pressure gradient. Journal of Fluid Mechanics, 877:1007-1062, 2019.

7. S. Orestano. Low-speed airfoil noise simulation - DNS post-processing on a controlleddiffusion airfoil. Master's thesis, École Centrale de Lyon, Écully, France, September 2019 .

8. G. A. Gerolymos, D. Sénéchal, and I. Vallet. Wall effects on pressure fluctuations in turbulent channel flow. Journal of Fluid Mechanics, 720:15-65, 2013.

9. R. H. Kraichnan. Pressure field within homogeneous anisotropic turbulence. Journal of the Acoustical Society of America, 28(1):64-72, January 1956.

10. T. H. Hodgson. Pressure fluctuations in shear flow turbulence. PhD thesis, The College of Aeronautics, Cranfield, 1961.

11. D. K. Wilson. Three-dimensional correlation and spectral functions for turbulent velocities in homogeneous and surface-blocked boundary layers. Technical report, Army Research Laboratory, July 1997.

12. D. K. Wilson. Turbulence models and the synthesis of random fields for acoustic wave propagation calculations. Technical report, Army Research Laboratory, July 1998.

13. Th. von Kármán. Progress in the statistical theory of turbulence. Proc. Nat. Acad. Sci., 34:530-539, 1948.

14. H. W. Liepmann, J. Laufer, and K. Liepmann. On the spectrum of isotropic turbulence. Technical report, National Advisory Committee for Aeronautics, November 1951.

15. J. C. R. Hunt. A theory of turbulent flow round two-dimensional bluff bodies. Journal of Fluid Mechanics, 61:625-706, 1973.

16. R. Schlinker and R. K. Amiet. Helicopter trailing edge noise. Technical report, NASA, 1981.

17. G. I. Taylor. The spectrum of turbulence. Proceedings of the Royal Society of London. Series A - Mathematical and Physical Sciences, 164(919):476 - 490, February 1938.

18. D. E. Hobbs and H. D. Weingold. Development of controlled diffusion airfoils for multistage compressor application. Journal of Engineering for Gas Turbines and Power, 106(2):271-278, 1984 .

19. S. Moreau. Symposium on the cd airfoil, https://www.researchgate.net/publication/ 304582435_CD-day_S-Moreau, June 2016. 
20. S. Moreau and M. Roger. Effect of airfoil aerodynamic load on trailing edge noise. AIAA Journal, 43(1):41-52, January 2005.

21. T. Padois, P. Laffay, A. Idier, and S. Moreau. Detailed experimental investigation of the aeroacoustic field around a Controlled-Diffusion airfoil. American Institute of Aeronautics and Astronautics, 2019/03/04 2015.

22. R. D. Sandberg. Compressible-flow DNS with application to airfoil noise. Flow, Turbulence and Combustion, 95:211-229, 2015.

23. Shiyi Chen and Gary D. Doolen. Lattice Boltzmann method for fluid flows. Annual Review of Fluid Mechanics, 30(1):329-364, January 1998.

24. $\mathrm{H}$. Wu, S. Moreau, and R. Sandberg. Effects of pressure gradient on the evolution of velocity gradient tensor invariant dynamics on a controlled-diffusion aerofoil at $R e_{c}=$ 150, 000. Journal of Fluid Mechanics, 868:584-610, February 2019.

25. S. Moreau, M. Sanjosé, F. Perot, and M.-S. Kim. Direct self-noise simulation of the installed controlled diffusion airfoil. In 17th AIAA/CEAS Aeroacoustics Conference (32nd AIAA Aeroacoustics Conference).

26. H. Wu, M. Sanjosé, S. Moreau, and R. D. Sandberg. Direct numerical simulation of the self-noise radiated by the installed Controlled-Diffusion airfoil at transitional Reynolds number. In 24th AIAA/CEAS Aeroacoustics Conference, AIAA Paper 2018-3797, Atlanta, Georgia, USA, 2018.

27. H. Wu, S. Moreau, and R. D. Sandberg. On the noise generated by a controlled-diffusion aerofoil at rec $=1.5 \times 105$. J. Sound Vib., 487:115620, 2020.

28. J. Magnaudet. High-reynolds-number turbulence in a shear-free boundary layer: revisiting the hunt-graham theory. Journal of Fluid Mechanics, 484:167-196, 2003.

29. M. Goody. Empirical spectral model of surface pressure fluctuations. AIAA Journal, 42(9):1788-1794, 2004.

30. Y. Rozenberg, G. Robert, and S. Moreau. Wall-pressure spectral model including the adverse pressure gradient effects. AIAA Journal, 50(10):2168-2179, 2012.

31. M. Sanjose, C. Meon, S. Moreau, A. Idier, and P. Laffay. Direct numerical simulation of acoustic reduction using serrated trailing-edge on an isolated airfoil. In 20th AIAA/CEAS Aeroacoustics Conference, Atlanta, GA, June 2014. American Institute of Aeronautics and Astronautics. 